\title{
Boundary element method application for the impressed current cathodic protection of an offshore platform
}

\author{
X. Wang ${ }^{1}$, B. Hou ${ }^{1} \&$ Z. Lan $^{2}$ \\ ${ }^{1}$ Institute of Oceanology, Chinese Academy of Sciences, China \\ ${ }^{2}$ CNOOC Research Institute, China
}

\begin{abstract}
For most steel structures in a marine environment, corrosion is a big problem which relates to long-term safety. Cathodic protection is an effective method to resist corrosion in seawater. In most cases, the design of cathodic protection systems depends on the empirical experience of the corrosion engineers. To achieve the protection potential of the whole structure, excess protective current is usually applied, which leads to the waste of protection material. In this paper, a boundary element method was used to simulate the impressed current cathodic protection of an offshore oil platform to achieve ideal potential and current distribution. The location and the amount of anodes with different current demand were calculated using the commercial software Beasy, which has many applications in the corrosion field. The boundary condition of the modelling is defined as the parameter of the anode and the cathode of the system, which can be illustrated with the polarization curve of the material in seawater. A physical model was also built to verify the numerical modelling results, and the experimental results were consistent with the computation prediction. The research proved the efficiency and accuracy of the boundary element method for the impressed current cathodic protection of the offshore structures.
\end{abstract}

Keywords: boundary element method, corrosion, impressed current cathodic protection, offshore platform.

\section{Introduction}

As the rapid growing demand of the energy, the offshore oil and gas exploitation has become an important assurance of energy supply. Cathodic protection is an effective technology to prevent the steel structure from the severe corrosion in 
marine environment. Generally the duty lifetime of the offshore oil platform is about 20 to 30 years, and the cathodic protection system is also designed for the same lifetime with the structure. However, in many marine oil exploiting fields, the offshore platform in duty usually exceeds its design life as the rich petroleum reservoir and the inaccurate prediction of the mining rate. According to the statistical data, $35 \%$ of the platforms with 20 years old need to continue to serve the production, which demand complete structure healthy evaluation and the necessary reinforcement to guarantee the structure safety. The cathodic protection system is one of the important parts of this reinforcement, and the repairing or renovating is needed for original system to accommodate the need of future duty life. On the other hand, for most of the offshore platform built 10 to 30 years ago, the sacrificial cathodic protection system was the most common method to resist the marine corrosion. The consumption rate of the anodes of the system sometimes exceeds the original design rate as the effect of marine environment factor, the poor anode quality, the inadequate anode current.

Though the impressed current cathodic protection (ICCP) and sacrificial anode cathodic protection are both used for the offshore structures, they have different features respectively. For those offshore structures which require extending or retrofitting the cathodic protection, impressed current system is an economic and simple choice which has great advantages over sacrificial anode system. Firstly, the large amount of galvanic anodes are usually installed on land before launching to the designated area, if all these anodes are retrofitted underwater, it would take great expense, while the ICCP can reduce most of the installation work. Secondly, as the structures surface has changed a lot comparing with the initial steel surface because of the calcareous deposit and the coating failure or breakage, it is difficult to evaluate the accurate current demand for protection, and once the amount and the location of the anodes has been determined, the current cannot be adjusted, while the ICCP can adjust the output current conveniently according to the monitored potential and current. So for the marine structures which need retrofitting, the ICCP is the optimal choice.

However, the selection of cathodic protection parameter is a large problem that the corrosion engineers concerned. For the long history during last several decades, the design depended on the experience of the engineers, especially for the design of the location and the amount of the anodes. With the dramatic advances of the numerical simulation analysis and the computing technology, the computer modeling method was used in the cathodic protection field, among which the boundary element method (BEM) has showed great advantages in the accuracy for the large marine structures. Comparing to other numerical analysis method, such as finite element method (FEM) [1,2], finite difference method (FDM), and the BEM analysis can effectively solve three dimension structure of large size with less mesh quantity [3-6].

In this paper, the Beasy software [7], which was developed based on boundary element method, was used to aid the design of ICCP system, and a jacket model was built in the seawater tank. The computing results and the experimental data were compared to check the validity of the numerical analysis. 


\section{Experimental}

\subsection{The ICCP principle}

Metal corrosion is an electrochemical reaction with oxidation and reduction reaction on different electrodes. For the steel in seawater, the following reactions occur:

$$
\begin{gathered}
\mathrm{Fe}_{(\mathrm{s})} \rightarrow \mathrm{Fe}_{(a q)}^{2+}+2 e^{-} \\
\mathrm{O}_{2(\mathrm{~g})}+2 \mathrm{H}_{2} \mathrm{O}_{(l)}+4 e^{-} \rightarrow 4 \mathrm{OH}_{(a q)}^{-} \\
2 \mathrm{Cl}^{-} \rightarrow \mathrm{Cl}_{2}+2 e^{-}
\end{gathered}
$$

In the reactions, Fe with solid state converts to Ferrous II ions with aqueous state, which means the loss of the steel. To inhibit the oxidation reaction, some measures have been taken to prevent the corrosion. For the cathodic protection, a type of metal with more negative potential or a power source connect with the steel and provide additional electrons. In the ICCP system of steel structure, a DC power (or rectifier converting $\mathrm{AC}$ power to $\mathrm{DC}$ power) and a type of nonconsumable anode are required. The principle of ICCP of steel structure in marine environment is depicted in fig. 1.

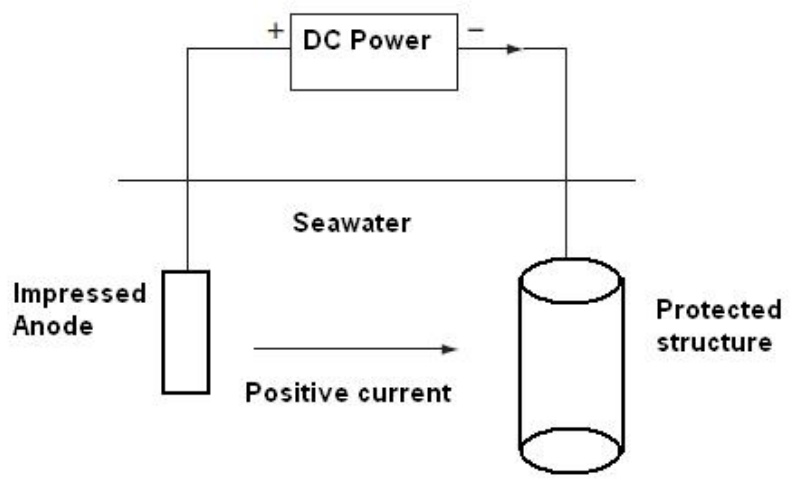

Figure 1: Diagram of impressed current cathodic protection.

In practice, the design of ICCP usually refers to some standards or criteria, such as NACE RP0176 and DNV RPB401. The most important point of the design is that the overprotection and under protection must be avoided. The commonly accepted protection potential is from $-850 \mathrm{mV}$ to $-1050 \mathrm{mV}$ vs. silver/silver chloride electrode.

The selection of proper protection potential and current density is very important which concerns about the final distribution of ICCP. The current density for ICCP is dependent on the environmental factors such as $\mathrm{pH}$ value, sanlinity, oxygen content, coating integrity, marine fouling, sea currents, pressure and calcareous deposit etc. 
The numerical simulation provides an effective method to help advance understanding the distribution of the potential and current density of the marine structures. As the offshore installation cost is very high, the simulation can modeling the real-time corrosion protection condition in advance to reduce the unreasonable problems caused by the design.

\subsection{ICCP experiment}

To simulate the cathodic protection condition of the marine structure, a physical model of jacket platform was scaling-down constructed referring to the real platform of China National Offshore Oil Corporation (CNOOC). The jacket platform had four legs and three layers in the vertical direction and had a symmetrical design. The legs and the support tubes of the platform have different diameter. The detailed dimension and structure of the platform was showed in fig. 2. The platform was made of steel without any coating on the surface. All the terminals of tubes were sealed to avoiding the entering of the seawater.

The platform was placed in the marine environment modeling tank, which was illustrated as the former research [8]. The modeling tank is large enough to place the jacket in. The accurate control system is designed to model the flow of the tide through a paddle wave maker and a siphon tube. The solution in the tank is fresh seawater pumped from the sea.

For the design of ICCP system of the marine structure, some factors are needed including seawater resistivity, total protective current required, the anode type and the design life.

The total current can be calculated by multiplying the current density and the exposed area of the structure.

The total current requirement $\left(I_{\text {req }}\right)$ for the offshore structure can be calculated by multiplying the total exposed area $(S)$ of the structure and the current density $\left(i_{j}\right)$ for protection, which can be described as below:

$$
I_{r e q}=i_{j} \cdot S
$$

After the total current has been decided, how to distribute the current to the whole structure is the major problem, so the arrangement of the anodes is very important.

To investigate the effect of the distance between anode and structure, the anode were placed at $20 \mathrm{~cm}$ and $60 \mathrm{~cm}$ distance from the platform respectively.

The electrochemical polarization experiment was conducted using a traditional three-electrode cell setup. The working electrode was steel with $1 \mathrm{~cm} \times 1 \mathrm{~cm} \times 1 \mathrm{~cm}$ dimension and the exposed area was $1 \mathrm{~cm}^{2}$. The reference electrode was saturated calomel electrode (SCE) with Luggin capillary, and the counter electrode was rectangular platinum sheet. The working electrode was abraded gradually using 600,800 and 1200 grit Sic paper, degreased by acetone, washed with distilled water and dried in air.

Electrochemical experiments were carried out using a Parstat 2273. The polarization curve measurement performed a scan starting from $-200 \mathrm{mV}$ to $+200 \mathrm{mV}$ vs. open circuit potential at a scan rate of $0.5 \mathrm{mV} / \mathrm{s}$. 


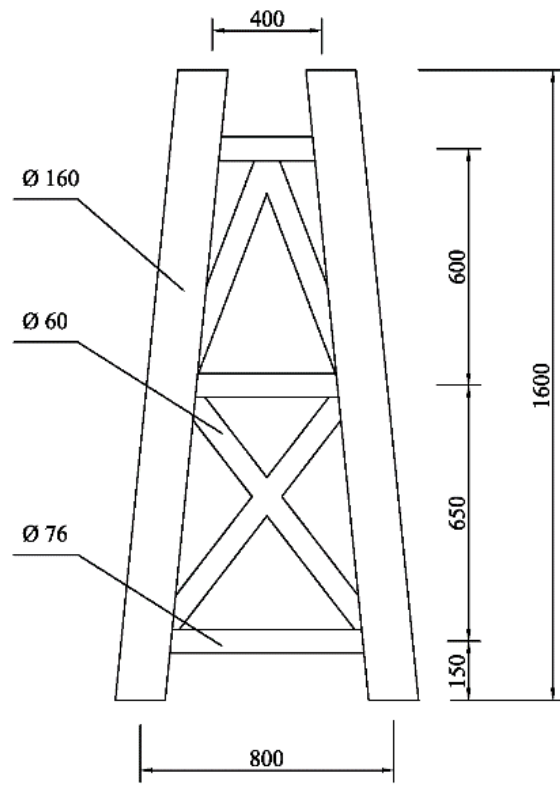

Front View

Figure 2: The detailed dimension of the platform (mm).

\section{Results and discussion}

\subsection{Boundary element method}

Boundary element method used for the corrosion computation is related to the Laplace equation [8]:

$$
\nabla^{2} \phi=0
$$

The Laplace equation can be solved using the boundary conditions illustrated in fig. 3 .

Some assumption simplifications are made for the corrosion conditions. $\Gamma$ is the entire surface of the electrolyte domain $\Omega$, which include $\Gamma_{1}, \Gamma_{2}, \Gamma_{3 a}$ and $\Gamma_{3 b}$. $\Phi$ and $I$ represent the potential and current density of the corresponding point. $\Phi_{0}$ and $I_{0}$ are initial constant values of potential and current density, respectively. $f_{a}\left(\varphi_{a}\right)$ and $f_{c}\left(\varphi_{c}\right)$ are relationships between potential and current density of anode and cathode respectively. For the ICCP system, the boundary condition parameter of anode is specified as constant potential or constant current value, and the cathode parameter can be measured using linear polarization method.

The Beasy software, which was designed for solving the Laplace equation and the corresponding computation matrix, has been used in corrosion field for several years. The key point of obtaining ideal computation result is that the construction of the model and the third boundary condition as described in fig. 3 . 


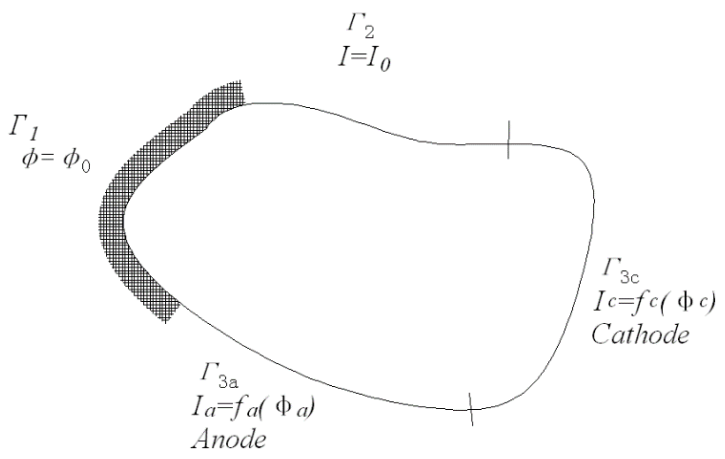

Figure 3: The boundary conditions for BEM.

\subsection{Numerical modeling}

The first step for the cathodic protection modeling is that the construction of the numerical model in graphical user interface. The model was built through the coordinate of every point in design drawing sheet. In the numerical model, all the dimension parameters were introduced into the Beasy software, including the cross support tubes in the vertical and horizontal direction, and the diameter dimension of every tube was also included. On this basis, the anode model was added, with the accurate dimension, shape, and the location on the model. The numerical model of the platform was showed in fig. 4 .

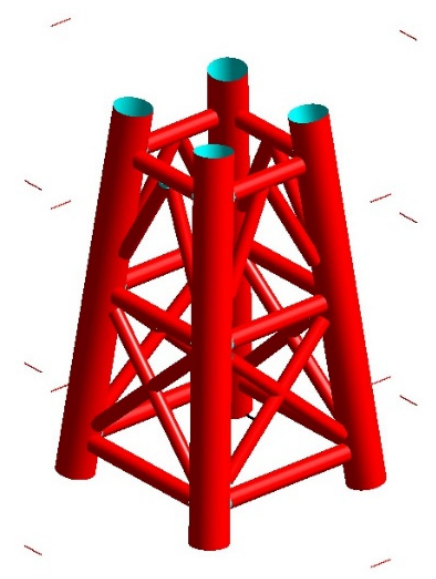

Figure 4: Numerical model of the platform.

After the accomplishment of the numerical model construction, the polarization data obtained using electrochemical experiment was digitized into the software polarization database, which was the third boundary condition for the computer modeling. Besides the parameters above, the environmental conditions of the 
seawater solution were also very important, such as the electrical conductivity and the temperature would affect the potential and current distribution of the cathodic protection system.

In this experiment, three impress current anodes were placed on each side of the jacket, a total of 12 anodes. To evaluate the influence of the distance between anode and jacket on the cathodic protection, different distance values were arranged.

The calculated results of the potential distribution of the jacket were described in fig. 5 . For the anodes with $20 \mathrm{~cm}$ distance from the jacket, the potential value

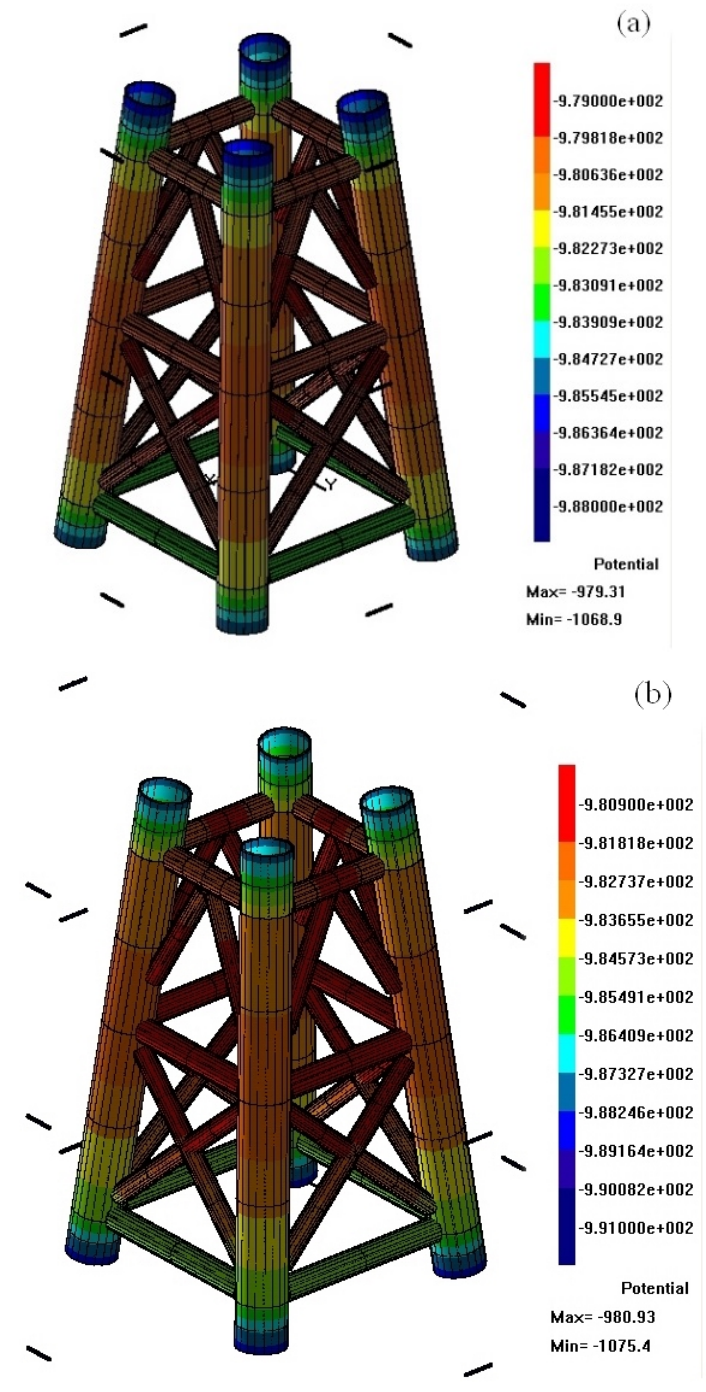

(a)

Figure 5: Potential distribution of the platform with ICCP (a) $20 \mathrm{~cm}$, (b) $60 \mathrm{~cm}$. 
was in the range of $-979.3 \mathrm{mV}$ to $-1068.9 \mathrm{mV}$, while the anodes with $60 \mathrm{~cm}$ distance from the jacket, the potential value range was $-980.9 \mathrm{mV}$ to $-1075.4 \mathrm{mV}$. The potential contour can visually reflect the potential distribution and the effect of anodes location and amount on the jacket. The real potential distribution on the platform was measured to verify the numerical results. For the 20 typical points on the platform, the results showed that the different between numerical modeling and measured results was less than $12 \mathrm{mV}$, which was small enough to prove the effectiveness of the boundary element method for ICCP modeling.

\section{Conclusions}

In this research, the potential distribution of the jacket with impressed current cathodic protection in seawater was modeling with boundary element method. The effect of the cathodic protection with different arrangements of the amount and location of the anodes was also compared. The BEM modeling has been proved feasible for the cathodic protection system of the offshore jacket.

\section{Acknowledgements}

The authors would acknowledge the financial support of the International S\&T Cooperation Program of China (2010DFR50860) and National Key Technology R\&D Program (Grant No. 2012BAB15B01).

\section{References}

[1] S.M. Sharland, C.P. Jackson, A.J. Diver, A finite-element model of the propagation of corrosion crevices and pits, Corrosion Science, 29(9), 1149-1166, 1989.

[2] C. Zhou, C. Wang, Y. Song, Evaluation of cyclic oxidation of thermal barrier coatings exposed to $\mathrm{NaCl}$ vapor by finite element method, Materials Science and Engineering: A, 490(1-2), 351-358, 2008.

[3] V.G. DeGiorgi, S.A. Wimmer, Geometric details and modeling accuracy requirements for shipboard impressed current cathodic protection system modeling, Engineering Analysis with Boundary Elements, 29(1), 15-28, 2005.

[4] Shigeru Aoki, Kenji Amaya, Optimization of cathodic protection system by BEM, Engineering Analysis with Boundary Elements, 19(2), 147-156, 1997.

[5] K. Amaya, S. Aoki, Effective boundary element methods in corrosion analysis, Engineering Analysis with Boundary Elements, 27(5), 507-519, 2003.

[6] S. Aoki, K. Kishimoto, Prediction of galvanic corrosion rates by the boundary element method, Mathematical and Computer Modelling, 15(3-5), 11-22, 1991.

[7] BEASY cathodic protection software user guide manual, version 10, CM Beasy limited.

[8] Z. Lan, X. Wang, B. Hou, Z. Wang, J. Song, S. Chen, Simulation of sacrificial anode protection for steel platform using boundary element method, Engineering Analysis with Boundary Elements, 36(6), 903-906, 2012. 